\title{
Decentralized Adaptive Control of Systems with Uncertain Interconnections, Plant-Model Mismatch and Actuator Failures
}

\author{
Parag Patre and Suresh M. Joshi
}

\begin{abstract}
Decentralized adaptive control is considered for systems consisting of multiple interconnected subsystems. It is assumed that each subsystem's parameters are uncertain and the interconnection parameters are not known. In addition, mismatch can exist between each subsystem and its reference model. A strictly decentralized adaptive control scheme is developed, wherein each subsystem has access only to its own state but has the knowledge of all reference model states. The mismatch is estimated online for each subsystem and the mismatch estimates are used to adaptively modify the corresponding reference models. The adaptive control scheme is extended to the case with actuator failures in addition to mismatch.
\end{abstract}

\section{INTRODUCTION}

The problem of controlling interconnected systems has been studied extensively over last four decades. It is of practical importance as it appears in various engineering applications such as power systems, aircraft formation flying, cooperative robotics, communication systems, etc. where the overall system is composed of several subsystems with dynamic interconnections or couplings. Usually, the objective is to design control inputs for the subsystems to track prespecified reference trajectories. Most of the research has focused on decentralized adaptive control [1], where the goal is to design an adaptive control for each subsystem using only the knowledge of its own state. This is desirable as it eliminates the adverse effects caused by communication delays and data losses between various subsystems. Often the structure of interconnections (coupling terms between different subsystem states) is assumed such that they lie in the range space of the input matrix [2], [3]. A highgain strategy with adaptive backstepping was used in [4] to relax the strict matching condition assumption, however, only bounded tracking is achieved. Some designs have relied on the bounds of these interconnections [5] to achieve partially decentralized adaptive schemes, and others have used Morse's dynamic certainty equivalence principle [6]. In [3] and [7], it was shown that it is theoretically possible to achieve asymptotic tracking in strictly decentralized adaptive control using the estimates of the unknown interconnections in the control input. However, strict decentralized adaptive

This research was supported in part by an appointment to the NASA Postdoctoral Program at Langley Research Center, administered by Oak Ridge Associated Universities through a contract with NASA.

Parag Patre is NASA Postdoctoral Program (NPP) Fellow at NASA Langley Research Center, Hampton, VA 23681, USA parag.patre@nasa.gov

Suresh M. Joshi is Senior Scientist for Control Theory at NASA Langley Research Center, Hampton, VA 23681, USA suresh.m.joshi@nasa.gov update laws could have large transient responses. A decisiontheoretic approach to decentralized adaptive control is presented in [8] that shows that there is improvement in transient performance when some communication is allowed between the subsystems .

The state-feedback-for-state-tracking (SFST) control architecture for model reference adaptive control (MRAC) relies on strict plant-model matching conditions to ensure stability and asymptotic tracking. The reference model design is usually based on the nominal plant model, and incorporates the desired closed-loop performance. Therefore, the nominal plant satisfies the matching condition. But the plant-model matching conditions could be violated due to abnormal changes in the plant or incorrect knowledge of the plant's mathematical structure [9]. In comparison to the previous results on decentralized adaptive control, dynamic systems consisting of interconnected subsystems with unknown interconnections are considered in this paper such that a plantmodel mismatch exists between the individual subsystems and their respective reference models. To compensate for the plant-model mismatch, the mismatch is estimated and is used to adaptively modify the reference model. The reference model is redesigned if the estimated plant-model mismatch exceeds a bound that is determined via robust stability and/or performance criteria. Because of the mismatch, the subsystems can no longer track the states of the original reference models, but may be able to track modified reference models that still provide satisfactory performance. To compensate for the unknown interconnections that are assumed to be in the range space of the control input matrix, their adaptive estimates are included in the control law similar to [3], [7]. The result is then extended to subsystems with multiple redundant actuators with actuator failures such that at least one actuator in each subsystem does not fail. A full-state feedback control law is designed for each subsystem using only its own state, while knowledge of all reference states in the adaptive laws is assumed (i.e., "implicit cooperation"). It is shown that the resulting control scheme offers asymptotic state tracking in the presence of plant-model mismatch, unknown interconnections, as well as actuator failures.

\section{Interconnected Systems With Plant-Model MISMATCH}

Consider a dynamic system $\Sigma$ consisting of $N$ interconnected subsystems $\Sigma_{i}, i=1,2, \ldots, N$, where each subsystem 
$\Sigma_{i}$ is described by

$$
\dot{x}_{i}(t)=\left(A_{0 i}+\delta a_{i}\right) x_{i}(t)+\sum_{j=1, j \neq i}^{N} A_{i j} x_{j}(t)+b_{i} u_{i}(t)
$$

where $x_{i}(t) \in \mathbb{R}^{n_{i}}$ is the subsystem state, $u_{i}(t) \in \mathbb{R}$ is the control input, $A_{0 i} \in \mathbb{R}^{n_{i} \times n_{i}}$ represents an known nominal system matrix, and $\delta a_{i} \in \mathbb{R}^{n_{i} \times n_{i}}$ represents the unknown constant parameter deviation in the system matrix. The control input matrix $b_{i} \in \mathbb{R}^{n_{i}}$ is assumed to be known. The constant matrices $A_{i j} \in \mathbb{R}^{n_{i} \times n_{j}}$ represent unknown interconnections between the subsystems $\Sigma_{i}$ and $\Sigma_{j}(j \neq i, j=1,2, \ldots, N)$.

Assumption 1: The unknown interconnections $A_{i j}$ are assumed to be in the range space of the control input matrix, i.e., they satisfy the matching condition

$$
A_{i j}=b_{i} l_{i j}^{T}
$$

where $l_{i j} \in \mathbb{R}^{n_{j}}$ are constant but unknown vectors with known bounds.

The structure of interconnections in (2) is identical to [2], [3] and guarantees the existence of a bounded control input $u_{i}(t)$ which can compensate for the unknown interconnections. Using the structure in (2), the dynamic system in (1) can be expressed as

$$
\dot{x}_{i}=\left(A_{0 i}+\delta a_{i}\right) x_{i}+b_{i}\left[\sum_{j=1, j \neq i}^{N} l_{i j}^{T} x_{j}+u_{i}\right] .
$$

\section{A. Control Objective}

There are $N$ reference models such that the $i^{\text {th }}$ reference model $\Sigma_{m i}$ is described by

$$
\dot{x}_{m i}(t)=A_{m i} x_{m i}(t)+b_{m i} r_{i}(t)
$$

where $x_{m i}(t) \in \mathbb{R}^{n_{i}}$ is the reference model state, $A_{m i} \in$ $\mathbb{R}^{n_{i} \times n_{i}}$ is an asymptotically stable matrix, $b_{m i} \in \mathbb{R}^{n_{i}}$, and $r_{i}(t) \in \mathbb{R}$ is a bounded reference input, chosen for some desired system behavior.

Assumption 2: The subsystem matrices $\left(A_{0 i}, b_{i}\right)$ and the reference models are assumed to satisfy the state-feedback for state-tracking (SFST) matching conditions, i.e., there exist (unknown) gains $k_{1 i} \in \mathbb{R}^{n_{i}}, k_{2 i} \in \mathbb{R}(i=1,2, \ldots, N)$ such that

$$
A_{m i}=A_{0 i}+b_{i} k_{1 i}^{T} ; \quad b_{m i}=b_{i} k_{2 i} .
$$

The description of the dynamic system in (3) is similar to the system in [3], however, in this paper we consider unknown parameter deviations $\delta a_{i}$ which represent unmatched uncertainties that do not satisfy the matching conditions for any values of $k_{1 i}, k_{2 i}$; therefore stability (signal boundedness) and asymptotic tracking are no longer guaranteed.

Assuming that each subsystem's controller has access only to its state $x_{i}(t)$ but has a complete knowledge of the reference states $x_{m i}(t)$ of all the reference models, the control objective is to design a strictly decentralized adaptive control, whose inputs $u_{i}(t) \in \mathbb{R}$ that will ensure closedloop signal boundedness and asymptotic tracking despite the unmatched uncertainty and unknown interconnections, i.e., $\lim _{t \rightarrow \infty}\left(x_{i}(t)-x_{m i}(t)\right)=0,(i=1,2, \ldots, N)$.

\section{B. Control Design}

The control input $u_{i}(t)$ of subsystem $\Sigma_{i}$ is designed as

$$
\begin{aligned}
u_{i}(t)= & \hat{k}_{1 i}^{T}(t) x_{i}(t)+\hat{k}_{2 i}(t) r_{i}(t) \\
& -\gamma_{i} e_{i}^{T}(t) P_{i} b_{i}-\sum_{j=1, j \neq i}^{N} \hat{l}_{i j}^{T}(t) x_{m j}(t)
\end{aligned}
$$

where $\hat{k}_{1 i}(t), \hat{k}_{2 i}(t), \hat{l}_{i j}(t)$ are the time-varying estimates of $k_{1 i}, k_{2 i}, l_{i j}$, respectively; $\gamma_{i} \in \mathbb{R}$ is a positive constant, $P_{i}=P_{i}^{T} \in \mathbb{R}^{n_{i} \times n_{i}}$ is a symmetric positive-definite matrix which is subsequently defined in (11), and $e_{i}(t) \in \mathbb{R}^{n_{i}}$ $(i=1,2, \ldots, N)$ are the subsystem tracking errors defined as

$$
e_{i}=x_{i}-x_{m i}
$$

In (6), the first two terms are typical in model reference adaptive control, the third term (similar to the high-gain term in [3], [5]) is used for the stability of the overall adaptive system, and the last term is used to cancel the effect of perturbations due to subsystems $\Sigma_{j}$.

Using (6) in (3), the $i^{\text {th }}$ closed-loop plant is given by:

$$
\begin{aligned}
\dot{x}_{i}= & \left(A_{0 i}+\delta a_{i}\right) x_{i}+b_{i}\left(\hat{k}_{1 i}^{T} x_{i}+\hat{k}_{2 i} r_{i}\right) \\
& -\gamma_{i} b_{i} e_{i}^{T} P_{i} b_{i}+b_{i} \sum_{j=1, j \neq i}^{N}\left[l_{i j}^{T} x_{j}-\hat{l}_{i j}^{T} x_{m j}\right] .
\end{aligned}
$$

Adding and subtracting $b_{i}\left(k_{1 i}^{T} x_{i}+k_{2 i} r_{i}\right)$ to (8), and using the SFST matching conditions (5) yields

$$
\begin{array}{r}
\dot{x}_{i}=\left(A_{m i}+\delta a_{i}\right) x_{i}+b_{m i} r_{i}+b_{i}\left(\tilde{k}_{1 i}^{T} x_{i}+\tilde{k}_{2 i} r_{i}\right) \\
-\gamma_{i} b_{i} e_{i}^{T} P_{i} b_{i}+b_{i} \sum_{j=1, j \neq i}^{N}\left[l_{i j}^{T} x_{j}-\hat{l}_{i j}^{T} x_{m j}\right] .
\end{array}
$$

where $\tilde{k}_{1 i}(t)=\hat{k}_{1 i}(t)-k_{1 i}$ and $\tilde{k}_{2 i}(t)=\hat{k}_{2 i}(t)-k_{2 i}$.

The above state equation indicates that it is not possible to follow the reference model (4). In order to accommodate plant-model mismatch and parameter deviation, the $i^{\text {th }}$ reference model is modified as $\dot{x}_{m i}=\left(A_{m i}+\widehat{\delta a}_{i}\right) x_{m i}+b_{m i} r_{i}$, where $\widehat{\delta a}_{i}(t)$ denotes the estimate of $\delta a_{i}$. Denote $A_{M i}(t)=$ $A_{m i}+\widehat{\delta a}_{i}(t)$, thus, the modified time-varying reference model is

$$
\dot{x}_{m i}=A_{M i} x_{m i}+b_{m i} r_{i} .
$$

This representation of the reference model indicates that the changed plant with mismatch can no longer follow the original reference model (4), but can possibly follow the modified time-varying reference model (10). The reference model depends on the mismatch parameter estimates, i.e., it "adapts" to the changed plant, and is therefore an "adaptive" reference model. However, it is necessary to first ensure the suitability of the the modified reference model, i.e., it must have acceptable stability and performance characteristics. Conventional MRAC schemes use a time-invariant reference model; therefore this time-varying reference model is a 
departure from standard MRAC. In the present approach, a time-invariant Lyapunov function weighting matrix is used along with a quadratic stability argument to ensure stability of the time-varying reference model. This can be accomplished by calculating the permissible limits on the estimated perturbations within which the stability and performance will remain satisfactory for all (time-varying) perturbations within these limits.

\section{Stability of Modified Reference Model}

The time-varying matrix $A_{M i}(t)$ can be expressed as an affine function of a parameter vector $\mathbf{p}_{i} \in R^{n_{p_{i}}}$ that lies in a convex polytope $\mathcal{S}_{i}$ having vertices $\mathbf{p}_{\mathbf{i}}^{\mathbf{k}_{\mathbf{i}}}, k_{i}=1, \ldots n_{v_{i}}$. For example, $A_{M i}(t)$ can be expressed as

$$
A_{M i}\left(\mathbf{p}_{\mathbf{i}}(t)\right)=A_{m i}+\sum_{q_{i}=1}^{n_{p_{i}}} \mathbf{p}_{\mathbf{i}_{\mathbf{q}_{\mathbf{i}}}}(t) A_{M i_{q_{i}}}
$$

where $A_{M i_{q_{i}}}$ are constant matrices and $\mathbf{p}_{\mathbf{i}_{\mathbf{q}_{\mathbf{i}}}}(t) \in\left[\mathbf{p}_{\mathbf{i}_{\mathbf{q}_{\mathbf{i}}}}, \overline{\mathbf{p}_{\mathbf{i}_{\mathbf{i}}}}\right]$, $\left(\mathbf{p}_{\mathbf{i}_{\mathbf{q}_{\mathbf{i}}}}(t)\right.$ is the $q_{i}$ th component of $\left.\mathbf{p}_{\mathbf{i}}\right)$. In this case $\mathcal{S}_{i}$ is a hyper-rectangular region with $n_{v_{i}}=2^{n_{p_{i}}}$ vertices. Let $A_{M i}\left(\mathbf{p}_{\mathbf{i}}^{\mathbf{k}_{\mathbf{i}}}\right)$ denote the value of the reference model system matrix at vertex $\mathbf{p}_{\mathbf{i}}^{\mathbf{k}_{\mathbf{i}}}$. Suppose there exist positive definite matrices $P_{i}=P_{i}^{T}, Q_{i}=Q_{i}^{T} \in R^{n_{i} \times n_{i}}$, such that

$$
A_{M i}\left(\mathbf{p}_{\mathbf{i}}^{\mathbf{k}_{\mathbf{i}}}\right)^{T} P_{i}+P_{i} A_{M i}\left(\mathbf{p}_{\mathbf{i}}^{\mathbf{k}_{\mathbf{i}}}\right)<-Q_{i}, k_{i}=1, \ldots n_{v_{i}}
$$

For a given $Q_{i},(11)$ represents a set of $n_{v_{i}}$ linear matrix inequalities (LMIs) in the unknown variable $P_{i}$. In view of (11), the Lyapunov inequality

$$
A_{M i}\left(\mathbf{p}_{\mathbf{i}}(t)\right)^{T} P_{i}+P_{i} A_{M i}\left(\mathbf{p}_{\mathbf{i}}(t)\right)<-Q_{i}
$$

is satisfied $\forall \mathbf{p}_{\mathbf{i}}(t) \in \mathcal{S}_{i}$, and the autonomous part of the reference model (i.e., the expression in (10) with $r_{i}=0$ ) is exponentially stable $\forall \mathbf{p}_{\mathbf{i}}(t) \in \mathcal{S}_{i}$ with a guaranteed minimum decay rate $e^{-\lambda_{\min }\left(P_{i}^{-1} Q_{i}\right) t}$. The solution $P_{i}=P_{i}^{T}$ to the Lyapunov inequality in (12) is used in the control input in (6). Express $\mathbf{p}_{\mathbf{i}_{\mathbf{q}_{\mathrm{i}}}}$ and $\overline{\mathbf{p}_{\mathbf{i}_{\mathbf{q}_{\mathrm{i}}}}}$ as

$$
\mathbf{p}_{\mathbf{i}_{\mathbf{q}_{\mathbf{i}}}}=\theta_{i} \underline{\delta_{i_{q_{i}}}} ; \quad \overline{\mathbf{p}_{\mathbf{i}_{\mathbf{q}_{\mathbf{i}}}}}=\theta_{i} \overline{\delta_{i q_{i}}} ; \quad q_{i}=1, \ldots n_{p_{i}}
$$

where $\underline{\delta_{i_{i}}}, \overline{\delta_{i_{q_{i}}}}$ are known constants (chosen to normalize the parameter ranges), and $\delta_{i_{q_{i}}} \leq 0 \leq \overline{\delta_{i_{q_{i}}}}$. The problem is to obtain the maximal region $\mathcal{S}_{i}$ for which (11) holds, i.e., find a positive definite symmetric matrix $P_{i}$ that maximizes $\theta_{i}$ subject to the set of LMIs in (11). The stability of the reference model depends only on $\widehat{\delta a}_{i}(t)$; therefore, if (11) is satisfied, the performance of the modified reference model would be acceptable. If $\widehat{\delta a}_{i}(t)$ approaches its permissible bounds, the reference model will need to be updated (redesigned) online.

\section{Adaptive Laws and Stability Analysis}

Using (9) and (10) in (7) and adding and subtracting $\delta a_{i} x_{i}$ to the resulting expression, we get the following closed-loop error system:

$$
\begin{aligned}
\dot{e}_{i}= & A_{M i} e_{i}-\widetilde{\delta a}_{i} x_{i}+b_{i}\left(\tilde{k}_{1 i}^{T} x_{i}+\tilde{k}_{2 i} r_{i}\right) \\
& -\gamma_{i} b_{i} e_{i}^{T} P_{i} b_{i}+b_{i} \sum_{j=1, j \neq i}^{N}\left[l_{i j}^{T} e_{j}-\tilde{l}_{i j}^{T} x_{m j}\right]
\end{aligned}
$$

where $\widetilde{\delta a}_{i}(t)=\widehat{\delta a}_{i}(t)-\delta a_{i}$ and $\tilde{l}_{i j}(t)=\hat{l}_{i j}(t)-l_{i j}$. For simplicity of presentation, we shall assume that $\widehat{\delta a}_{i}(t)$ remains within the quadratic stability bounds, however, as is common in literature, a simple parameter projection algorithm [9], [10] can be used to ensure that this condition is met.

Theorem 1: For the system given by (1), (10), the adaptive controller (6) with the gain adaptive laws

$$
\dot{\hat{k}}_{1 i}=-\Gamma_{1 i} x_{i} e_{i}^{T} P_{i} b_{i}, \dot{\hat{k}}_{2 i}=-\Gamma_{2 i} b_{i}^{T} P_{i} e_{i} r_{i}^{T}
$$

and parameter estimation laws

$$
\begin{aligned}
\dot{\widehat{\delta a}}_{i} & =-\Gamma_{a i} P_{i} e_{i} x_{i}^{T} \\
\dot{\hat{l}}_{i j} & =\Gamma_{l_{i j}} x_{m j} e_{i}^{T} P_{i} b_{i}, \quad(j \neq i, j=1,2, \ldots, N)
\end{aligned}
$$

where $\Gamma_{1 i} \in \mathbb{R}^{n_{i} \times n_{i}}, \Gamma_{2 i} \in \mathbb{R}, \Gamma_{a i} \in \mathbb{R}^{n_{i} \times n_{i}}, \Gamma_{l_{i j}} \in$ $\mathbb{R}^{n_{j} \times n_{j}}$ are symmetric positive definite matrices, guarantee that all closed-loop signals including adaptive gains, parameter estimates and interconnection estimates are bounded and the tracking error $e_{i}(t) \rightarrow 0$ as $t \rightarrow \infty, \quad(i=1,2, \ldots, N)$.

Proof: Define a Lyapunov function candidate for the whole system $\Sigma$ as

$$
\begin{aligned}
V= & \sum_{i=1}^{N}\left[e_{i}^{T} P_{i} e_{i}+\tilde{k}_{1 i}^{T} \Gamma_{1 i}^{-1} \tilde{k}_{1 i}+\Gamma_{2 i}^{-1} \tilde{k}_{2 i}^{2}\right] \\
& +\sum_{i=1}^{N}\left[\sum_{k=1}^{n_{i}} \widetilde{\delta a}_{i k}^{T} \Gamma_{a i}^{-1} \widetilde{\delta a}_{i k}+\sum_{j=1, j \neq i}^{N} \tilde{l}_{i j}^{T} \Gamma_{l_{i j}}^{-1} \tilde{l}_{i j}\right]
\end{aligned}
$$

where the subscript $k$ denotes the $k^{\text {th }}$ column of $\widetilde{\delta a_{i}}$. Differentiating (17) with respect to time, and using (14), leads to

$$
\begin{aligned}
\dot{V}= & \sum_{i=1}^{N}\left[2 e _ { i } ^ { T } P _ { i } \left\{A_{M i} e_{i}+b_{i}\left(\tilde{k}_{1 i}^{T} x_{i}+\tilde{k}_{2 i} r_{i}\right)\right.\right. \\
& \left.-\widetilde{\delta a_{i}} x_{i}-\gamma_{i} b_{i} e_{i}^{T} P_{i} b_{i}+b_{i} \sum_{j=1, j \neq i}^{N}\left[l_{i j}^{T} e_{j}-\tilde{l}_{i j}^{T} x_{m j}\right]\right\} \\
& +2 \tilde{k}_{1 i}^{T} \Gamma_{1 i}^{-1} \dot{\hat{k}}_{1 i}+2 \tilde{k}_{2 i} \Gamma_{2 i}^{-1} \dot{\hat{\hat{k}}} 2 i \\
& \left.+2 \operatorname{Tr}\left[\widetilde{\delta a}_{i}^{T} \Gamma_{a i}^{-1} \dot{\widehat{\delta a}}_{i}\right]+\sum_{j=1, j \neq i}^{N} 2 \tilde{l}_{i j}^{T} \Gamma_{l_{i j}}^{-1} \dot{\hat{l}}_{i j}\right] .
\end{aligned}
$$

The adaptive laws for $\hat{k}_{1 i}, \hat{k}_{2 i}, \widehat{\delta}_{i}$, and $\hat{l}_{i j}$, in (15) and (16) are substituted to yield

$$
\begin{aligned}
\dot{V} \leq & \sum_{i=1}^{N}\left[-\lambda_{\min }\left(Q_{i}\right)\left\|e_{i}\right\|^{2}-2 \gamma_{i}\left(e_{i}^{T} P_{i} b_{i}\right)^{2}\right] \\
& +\sum_{i=1}^{N} \sum_{j=1, j \neq i}^{N} 2 e_{i}^{T} P_{i} b_{i} l_{i j}^{T} e_{j}
\end{aligned}
$$


where $\lambda_{\min }\left(Q_{i}\right)$ is the smallest eigenvalue of $Q_{i}$. From (18), a sufficient condition to make $\dot{V}(t)$ negative-semidefinite can be derived as

$$
\gamma_{i}>\frac{1}{2}(N-1)^{2} \max _{j}\left(\frac{\left\|l_{i j}\right\|^{2}}{\lambda_{\min }\left(Q_{j}\right)}\right) .
$$

That is, $V(t)$ is bounded for all $t$, and standard stability analysis arguments [9] can be used to show that all signals and estimates are bounded, and $\lim _{t \rightarrow \infty}\left(x_{i}(t)-x_{m i}(t)\right)=$ $0,(i=1,2, \ldots, N)$.

\section{E. Reference Model Redesign/Update}

The adaptive reference model proposed in this paper has two levels of adaptation. At the first level, the time-varying reference model $A_{M i}(t), b_{m i}$ addresses relatively smallmagnitude mismatch (bounded by quadratic stability or performance bounds). The second level of adaptation addresses large magnitude mismatch; i.e., the reference model is updated (redesigned) when $\widehat{\delta a}_{i}(t)$ exceeds a predetermined threshold. This is expected to occur occasionally as finite number of discrete events, and the adaptive control scheme can be implemented as follows:

1) A reference model $A_{m i}, b_{m i}$ is designed based on the initial approximate knowledge of the plant $\left(A_{0 i}, b_{i}\right)$. The matching conditions (5) are satisfied.

2) Permissible limits on the elements of $\widehat{\delta a}_{i}(t)$ for quadratic stability (or other additional performance criteria) are computed.

3) Adaptive control law and mismatch estimation equations (6), (15), (16) are applied.

4) If any elements of $\widehat{\delta a}_{i}(t)$ approach the limits obtained in Step 2 (e.g., within 80 per cent), the system parameter estimates are updated by replacing $A_{0 i}$ by $A_{0 i}+\widehat{\delta a}_{i}(t)$, and the reference model is redesigned.

5) $\widehat{\delta a}_{i}(t)$ is set to zero, and the procedure returns to Step 2.

Steps 2 and 4 are performed real-time whenever a reference model redesign is required. This is feasible because of the availability of highly efficient numerical algorithms and the trend toward rapidly accelerating computational capability. To further ensure that the mismatch estimates remain within the permissible bounds, a projection algorithm (e.g., [9]) can be used.

\section{Simultaneous Model Mismatch And Actuator FAILURES}

In addition to model mismatch, the actuators (e.g., control surfaces in aircraft flight control) may fail during the operation. We consider a system such that each subsystem $\Sigma_{i}$ has multiple redundant actuators of the same type:

$$
\dot{x}_{i}(t)=\left(A_{0 i}+\delta a_{i}\right) x_{i}(t)+\sum_{j=1, j \neq i}^{N} A_{i j} x_{j}(t)+B_{i} u_{i}(t)
$$

where $u_{i}(t) \in R^{m_{i}}$ represents a group of similar actuators such that at least one actuator remains functional. In this case, the columns $b_{i_{f}} \in R^{n_{i}},\left(f=1,2, \ldots, m_{i}\right)$ of the known $B_{i} \in R^{n_{i} \times m_{i}}$ matrix are parallel to the reference model input matrix $b_{m i} \in R^{n_{i}}, b_{i_{f}}=b_{m i} / \alpha_{i_{f}}$ for some known $\alpha_{i_{f}}$ 's. The result can be easily extended for the case of unknown $\alpha_{i_{f}}$ (see [9]). The actuator failures are modeled in this paper as

$$
\begin{aligned}
u_{i_{f}}(t) & =\bar{u}_{i_{f}}, t \geq t_{i_{f}}, \\
f & \in\left\{f_{1}, f_{2}, \ldots, f_{p}\right\} \subseteq\left\{1,2, \ldots, m_{i}\right\}
\end{aligned}
$$

where the failure pattern $\left\{f_{1}, f_{2}, \ldots, f_{p}\right\}$, the failure value $\bar{u}_{i_{f}}$ (assumed to be constant), and the failure time of occurrence $t_{i_{f}}$ are all unknown. Let $v_{i}(t) \in R^{m_{i}}$ be the applied (commanded) control input vector. In the presence of actuator failures, the actual input vector $u_{i}(t)$ to the system can be described as

$$
\begin{aligned}
u_{i}(t) & =v_{i}(t)+\sigma_{i}\left(\bar{u}_{i}-v_{i}(t)\right) \\
& =\left(I-\sigma_{i}\right) v_{i}(t)+\sigma_{i} \bar{u}_{i}
\end{aligned}
$$

where

$$
\begin{aligned}
\bar{u}_{i} & =\left[\bar{u}_{i_{1}}, \bar{u}_{i_{2}}, \ldots, \bar{u}_{i_{m_{i}}}\right]^{T} \\
\sigma_{i} & =\operatorname{diag}\left\{\sigma_{i_{1}}, \sigma_{i_{2}}, \ldots, \sigma_{i_{m_{i}}}\right\} \\
\sigma_{i_{f}} & =1 \text { if the } f^{\text {th }} \text { actuator fails, i.e., } u_{i_{f}}=\bar{u}_{i_{f}} \\
\sigma_{i_{f}} & =0 \text { otherwise. }
\end{aligned}
$$

That is, $\sigma_{i} \in R^{m_{i} \times m_{i}}$ is a diagonal matrix whose entries are piecewise step- or zero- functions of time. The actuator failures are uncertain in value, pattern and time of occurrence. The objective of the adaptive controller is to synthesize the control signal $v_{i}(t)$ using only the knowledge of its state $x_{i}(t)$ and the desired states of all reference models, i.e., $x_{m j}(j=1,2, \ldots, N)$, so as to ensure the system stability and asymptotic tracking regardless of whether (or which) actuators have failed, or the failure values.

The unknown interconnections $A_{i j}$ in (20) are assumed to satisfy the matching condition $A_{i j}=B_{i}\left(I-\sigma_{i}\right) L_{i j}^{T}$, where $L_{i j} \in R^{n_{j} \times m_{i}}$ are unknown constant matrices.

The adaptive control input is designed as:

$$
v_{i}=\hat{K}_{1 i}^{T} x_{i}+\hat{k}_{2 i} r_{i}+\hat{k}_{3 i}-\gamma_{i} B_{i}^{T} P_{i} e_{i}-\sum_{j=1, j \neq i}^{N} \hat{L}_{i j}^{T} x_{m j}
$$

where $\gamma_{i} \in R$ is a constant scalar gain; $\hat{K}_{1} \in R^{n_{i} \times m_{i}}$, and $\hat{k}_{2 i}, \hat{k}_{3 i} \in R^{m_{i}}$ are time-varying adaptive gains; and $\hat{L}_{i j} \in R^{n_{j} \times m_{i}}$ are time-varying adaptive estimates of the interconnections. The control design in (23) is similar to (6) except for the third term $\hat{k}_{3 i}(t)$, which is included to compensate the effect of constant actuator failures. The $i^{\text {th }}$ closed-loop plant becomes

$$
\begin{aligned}
\dot{x}_{i}= & \left(A_{0 i}+\delta a_{i}\right) x_{i}+B_{i}\left(I-\sigma_{i}\right) v_{i} \\
& +B_{i} \sigma_{i} \bar{u}_{i}+\sum_{j=1, j \neq i}^{N} B_{i}\left(I-\sigma_{i}\right) L_{i j}^{T} x_{j}
\end{aligned}
$$


which upon substituting (23) yields

$$
\begin{aligned}
\dot{x}_{i}= & \left(A_{0 i}+\delta a_{i}+B_{i}\left(I-\sigma_{i}\right) K_{1 i}^{T}\right) x_{i} \\
& +B_{i}\left(I-\sigma_{i}\right) \tilde{K}_{1 i}^{T} x_{i}+B_{i}\left(I-\sigma_{i}\right) k_{2 i} r_{i} \\
& +B_{i}\left(I-\sigma_{i}\right) \tilde{k}_{2 i} r_{i}+B_{i}\left(I-\sigma_{i}\right) k_{3 i} \\
& +B_{i}\left(I-\sigma_{i}\right) \tilde{k}_{3 i}+B_{i} \sigma_{i} \bar{u}_{i}-\gamma_{i} B_{i}\left(I-\sigma_{i}\right) B_{i}^{T} P_{i} e_{i} \\
& +B_{i}\left(I-\sigma_{i}\right) \sum_{j=1, j \neq i}^{N}\left[L_{i j}^{T} x_{j}-\hat{L}_{i j}^{T} x_{m j}\right] .
\end{aligned}
$$

There can be up to $\left(m_{i}-1\right)$ actuator failures for each $\Sigma_{i}$. We assume that the following matching conditions are satisfied for the nominal plant $\left(A_{0 i}, B_{i}\right)$ for some $K_{1 i}, k_{2 i}$, and $k_{3 i}$, and for all actuator failure patterns $\left(\sigma_{i}\right)$.

$$
\begin{aligned}
A_{0 i}+B_{i}\left(I-\sigma_{i}\right) K_{1 i} & =A_{m i}, B_{i}\left(I-\sigma_{i}\right) k_{2 i}=b_{m i} \\
B_{i}\left(I-\sigma_{i}\right) k_{3 i} & =-B_{i} \sigma_{i} \bar{u}_{i} .
\end{aligned}
$$

Therefore, (24) can be expressed as

$$
\begin{aligned}
\dot{x}_{i}= & \left(A_{m i}+\delta a_{i}\right) x_{i}+b_{m i} r_{i}-\gamma_{i} B_{i}\left(I-\sigma_{i}\right) B_{i}^{T} P_{i} e_{i} \\
& +B_{i}\left(I-\sigma_{i}\right)\left(\tilde{K}_{1 i}^{T} x_{i}+\tilde{k}_{2 i} r_{i}+\tilde{k}_{3 i}\right) \\
& +B_{i}\left(I-\sigma_{i}\right) \sum_{j=1, j \neq i}^{N}\left[L_{i j}^{T} e_{j}-\tilde{L}_{i j}^{T} x_{m j}\right] .
\end{aligned}
$$

To accommodate the plant-model mismatch, the modified reference model in (10) is employed. Thus, the error equation is

$$
\begin{aligned}
\dot{e}_{i}= & A_{M i} e_{i}-\widetilde{\delta a}_{i} x_{i}-\gamma_{i} B_{i}\left(I-\sigma_{i}\right) B_{i}^{T} P_{i} e_{i} \\
& +B_{i}\left(I-\sigma_{i}\right)\left(\tilde{K}_{1 i}^{T} x_{i}+\tilde{k}_{2 i} r_{i}+\tilde{k}_{3 i}\right) \\
& +B_{i}\left(I-\sigma_{i}\right) \sum_{j=1, j \neq i}^{N}\left[L_{i j}^{T} e_{j}-\tilde{L}_{i j}^{T} x_{m j}\right] .
\end{aligned}
$$

Theorem 2: For the system given by (10) and (24), the control law (23) with gain adaptation laws

$$
\begin{aligned}
\dot{\hat{K}}_{1 i_{f}} & =-\Gamma_{1 i_{f}} x_{i} e_{i}^{T} P_{i} b_{i_{f}}, \dot{\hat{k}}_{2 i_{f}}=-\gamma_{2 i_{f}} b_{i_{f}}^{T} P_{i} e_{i} r_{i}^{T} \\
\dot{\hat{k}}_{3 i_{f}} & =-\gamma_{3 i_{f}} b_{i_{f}}^{T} P_{i} e_{i}
\end{aligned}
$$

and parameter estimation law

$$
\dot{\hat{\delta a}}_{i}=-\Gamma_{a i} P_{i} e_{i} x_{i}^{T}, \quad \dot{\hat{L}}_{i j_{f}}=\Gamma_{L_{i j_{f}}} x_{m j} e_{i}^{T} P_{i} b_{i_{f}}
$$

for $f=1, \ldots, m_{i}$ and $(j \neq i, j=1,2, \ldots, N)$ guarantee that all closed-loop signals including adaptive gains and parameter estimates are bounded and the tracking error $e_{i}(t) \rightarrow 0$ as $t \rightarrow \infty, \quad(i=1,2, \ldots, N)$.

Outline of proof- The expression in (27) can be written as

$$
\begin{aligned}
\dot{e}_{i}= & A_{M i} e_{i}-\widetilde{\delta a}_{i} x_{i}-\gamma_{i} B_{i}\left(I-\sigma_{i}\right) B_{i}^{T} P_{i} e_{i} \\
& +\sum_{f \notin \mathcal{F}_{p}}^{m_{i}} b_{i_{f}}\left(\tilde{K}_{1 i_{f}}^{T} x_{i}+\tilde{k}_{2 i_{f}} r_{i}+\tilde{k}_{3 i_{f}}\right) \\
& +\sum_{f \notin \mathcal{F}_{p}}^{m_{i}} \sum_{j=1, j \neq i}^{N} b_{i_{f}}\left[L_{i j_{f}}^{T} e_{j}-\tilde{L}_{i j_{f}}^{T} x_{m j}\right]
\end{aligned}
$$

where $\mathcal{F}_{p}=f_{1}, f_{2}, \ldots, f_{p}$ denotes the set of indices corresponding to the $p \leq m-1$ failed actuators. Define

$$
\begin{aligned}
V= & \sum_{i=1}^{N}\left[e_{i}^{T} P_{i} e_{i}+\sum_{k=1}^{n_{i}} \widetilde{\delta a}_{i_{k}}^{T} \Gamma_{a i}^{-1} \widetilde{\delta a}_{i_{k}}\right. \\
& +\sum_{f \notin \mathcal{F}_{p}}^{m_{i}}\left(\tilde{K}_{1 i_{f}}^{T} \Gamma_{1 i_{f}}^{-1} \tilde{K}_{1 i_{f}}+\tilde{k}_{2 i_{f}}^{2} \gamma_{2 i_{f}}^{-1}+\tilde{k}_{3 i_{f}}^{2} \gamma_{3 i_{f}}^{-1}\right) \\
& \left.+\sum_{f \notin \mathcal{F}_{p}}^{m_{i}} \sum_{j=1, j \neq i}^{N} \tilde{L}_{i j_{f}}^{T} \Gamma_{L_{i j_{f}}}^{-1} \tilde{L}_{i j_{f}}\right]
\end{aligned}
$$

Differentiating (31) with respect to time, and using (30) as well as properties of trace of a matrix, the following expression is obtained:

$$
\begin{aligned}
\dot{V}= & \sum_{i=1}^{N}\left[2 e _ { i } ^ { T } P _ { i } \left\{A_{M i} e_{i}-\gamma_{i} B_{i}\left(I-\sigma_{i}\right) B_{i}^{T} P_{i} e_{i}\right.\right. \\
& -\widetilde{\delta a_{i}} x_{i}+\sum_{f \notin \mathcal{F}_{p}}^{m_{i}} b_{i_{f}}\left(\tilde{K}_{1 i_{f}}^{T} x_{i}+\tilde{k}_{2 i_{f}} r_{i}+\tilde{k}_{3 i_{f}}\right) \\
& \left.+\sum_{f \notin \mathcal{F}_{p}}^{m_{i}} \sum_{j=1, j \neq i}^{N} b_{i_{f}}\left[L_{i j_{f}}^{T} e_{j}-\tilde{L}_{i j_{f}}^{T} x_{m j}\right]\right\} \\
& +2 \sum_{f \notin \mathcal{F}_{p}}^{m_{i}}\left(\tilde{K}_{1 i_{f}}^{T} \Gamma_{1 i}^{-1} \dot{\hat{K}}_{1 i_{f}}+\tilde{k}_{2 i} \gamma_{2 i_{f}}^{-1} \dot{\hat{k}}_{2 i}\right. \\
& \left.+\tilde{k}_{3 i_{f}} \gamma_{3 i_{f}}^{-1} \dot{\hat{k}}_{3 i_{f}}\right)+2 T r\left[\widetilde{\delta a}_{i}^{T} \Gamma_{a i}^{-1} \dot{\hat{\delta a}}_{i}\right] \\
& \left.+\sum_{f \notin \mathcal{F}_{p}}^{m_{i}} \sum_{j=1, j \neq i}^{N} 2 \tilde{L}_{i j_{f}}^{T} \Gamma_{l_{i j}}^{-1} \dot{\hat{L}}_{i j_{f}}\right] .
\end{aligned}
$$

Using the fact that

$$
2 \gamma_{i} e_{i}^{T} P_{i} B_{i}\left(I-\sigma_{i}\right) B_{i}^{T} P_{i} e_{i}=2 \gamma_{i} \sum_{f \notin \mathcal{F}_{p}}^{m_{i}}\left(e_{i}^{T} P_{i} b_{i_{f}}\right)^{2}
$$

and the adaptive update laws in (28) and (29), $\dot{V}(t)$ can be upper bounded as

$$
\begin{aligned}
\dot{V} \leq & \sum_{i=1}^{N}\left[-\lambda_{\min }\left(Q_{i}\right)\left\|e_{i}\right\|^{2}-2 \gamma_{i} \sum_{f \notin \mathcal{F}_{p}}^{m_{i}}\left(e_{i}^{T} P_{i} b_{i_{f}}\right)^{2}\right. \\
& \left.+\sum_{f \notin \mathcal{F}_{p}}^{m_{i}} \sum_{j=1, j \neq i}^{N} 2 b_{i_{f}} e_{i}^{T} P_{i} L_{i j_{f}}^{T} e_{j}\right] .
\end{aligned}
$$

From (32), a sufficient condition to make $\dot{V}(t)$ negative semi-definite can be derived as

$$
\gamma_{i}>\frac{1}{2} m_{i}(N-1)^{2} \max _{j}\left(\frac{\left\|l_{i j}\right\|^{2}}{\lambda_{\min }\left(Q_{j}\right)}\right) .
$$

The remainder of the proof is similar to that of Theorem 1 . 


\section{NUMERICAL EXAMPLE}

Two interconnected second-order subsystems of the form (20) are considered such that each has two identical actuators:

$$
\begin{aligned}
A_{01} & =\left[\begin{array}{cc}
2 & -2 \\
7 & 2
\end{array}\right], \delta a_{1}=\left[\begin{array}{cc}
0.45 & 0 \\
0 & 0
\end{array}\right], L_{12}=\left[\begin{array}{cc}
2 & -1 \\
3 & 7
\end{array}\right], \\
B_{1} & =\left[\begin{array}{cc}
0 & 0 \\
-1 & -1
\end{array}\right], \\
A_{02} & =\left[\begin{array}{cc}
0 & 4 \\
13 & 2
\end{array}\right], \delta a_{2}=\left[\begin{array}{cc}
0 & 0 \\
-1 & 1
\end{array}\right], L_{21}=\left[\begin{array}{cc}
-1 & 2 \\
5 & -4
\end{array}\right], \\
B_{2} & =\left[\begin{array}{ll}
3 & 3 \\
0 & 0
\end{array}\right]
\end{aligned}
$$

and the reference models:

$$
\begin{aligned}
& A_{m 1}=\left[\begin{array}{cc}
2 & -2 \\
15.06 & -6.04
\end{array}\right], B_{m 1}=B_{1} \\
& A_{m 2}=\left[\begin{array}{cc}
-16.82 & -6.81 \\
13 & 2
\end{array}\right], B_{m 2}=B_{2} .
\end{aligned}
$$

The states are

$$
x_{1}^{T}=\left[\begin{array}{ll}
x_{11} & x_{12}
\end{array}\right]^{T}, x_{2}^{T}=\left[\begin{array}{ll}
x_{21} & x_{22}
\end{array}\right]^{T},
$$

and the control inputs are

$$
u_{1}^{T}=\left[\begin{array}{ll}
u_{11} & u_{12}
\end{array}\right]^{T}, u_{2}^{T}=\left[\begin{array}{ll}
u_{21} & u_{22}
\end{array}\right]^{T} .
$$

The tracking performance for reference step input commands $r_{1}=5$ and $r_{2}=5$ starting at $t=1 \mathrm{~s}$ and $t=5 \mathrm{~s}$, respectively, is shown in Figs. 1-3 such that $u_{11}$ fails at $t=10$ s (i.e., it gets stuck at its current position at $t=10 \mathrm{~s}$ ). The parameter estimate $\widehat{\delta a_{12}}$ exceeds the safe bound 0.57 at $t=6.14 \mathrm{~s}$ causing a parameter reset and an update of the reference model. A new safe bound is calculated and is not exceeded for the rest of the run.

Fig. 1. Plant and reference model states
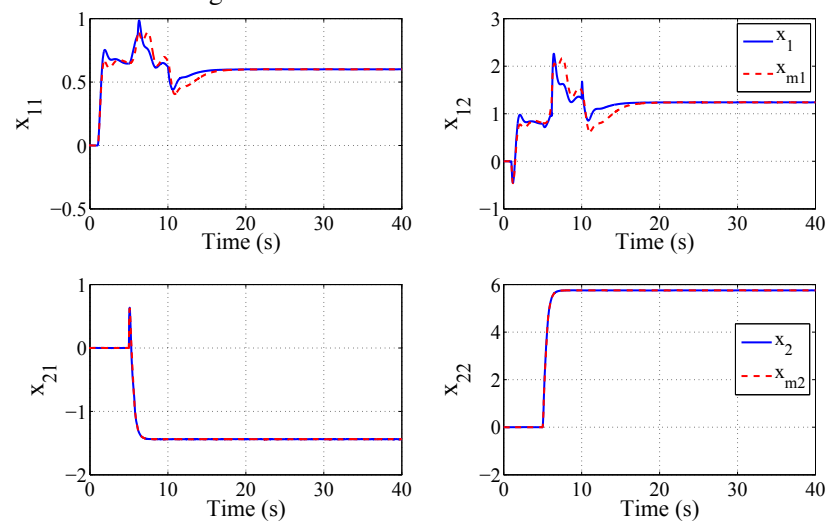

\section{CONCLUSION}

A decentralized adaptive control scheme was proposed for uncertain interconnected systems. The proposed adaptive controller can compensate for unknown interconnections between subsystems, as well as mismatch between each subsystem and its reference model. The scheme was extended to systems with actuator failures, and simulation results were presented for an example system.
Fig. 2. Control inputs
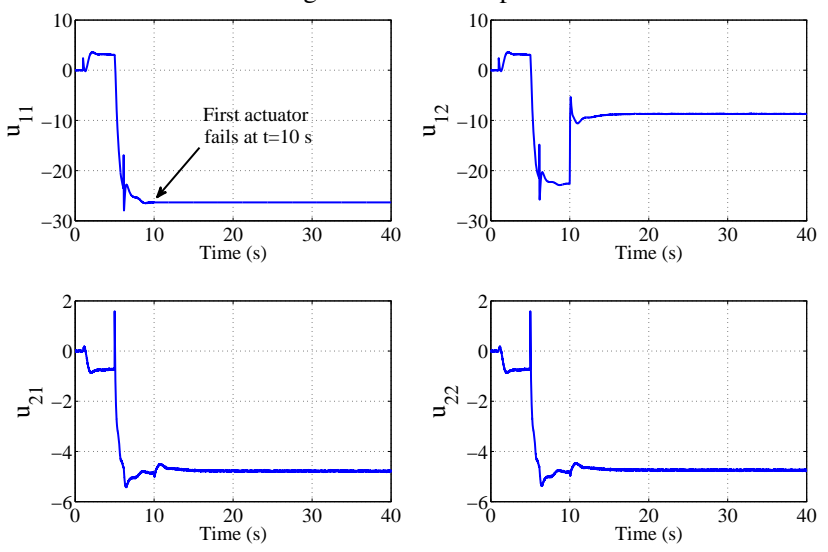

Fig. 3. Parameter estimates


\section{REFERENCES}

[1] P. Ioannou, "Decentralized adaptive control of interconnected systems," IEEE Trans. Automat. Contr., vol. 31, no. 4, pp. 291-298, 1986.

[2] D. T. Gavel and D. D. Siljak, "Decentralized adaptive control: structural conditions for stability," IEEE Trans. Automat. Contr., vol. 34, no. 4, pp. 413-426, 1989.

[3] K. S. Narendra and N. O. Oleng, "Exact output tracking in decentralized adaptive control systems," IEEE Trans. Automat. Contr., vol. 47, no. 2, pp. 390-395, 2002.

[4] S. Jain and F. Khorrami, "Decentralized adaptive control of a class of large-scale interconnected nonlinear systems," IEEE Trans. Automat. Contr., vol. 42, no. 2, pp. 136-154, 1997.

[5] L. Shi and S. K. Singh, "Decentralized adaptive controller design for large-scale systems with higher order interconnections," IEEE Trans. Automat. Contr., vol. 37, no. 8, pp. 1106-1118, 1992.

[6] R. Ortega and A. Herrera, "A solution to the decentralized adaptive stabilization problem," in Proc. IEEE Conf. Decision and Control, 1992, pp. 3622-3627.

[7] B. M. Mirkin, "Decentralized adaptive controller with zero residual tracking errors," in Proc. Mediterranean Conf. on Control and Autom., Haifa, Israel, June 1999, pp. 388-398.

[8] K. S. Narendra and S. Mukhopadhyay, "To communicate or not to communicate: A decision-theoretic approach to decentralized adaptive control," in Proc. Amer. Control Conf., Baltimore, MD, 2010, pp. 6369-6376.

[9] S. Joshi, G. Tao, and P. Patre, "Direct adaptive control using an adaptive reference model," Int. J. of Control, vol. 84, no. 1, pp. 180196, 2011.

[10] M. Krstic, I. Kanellakopoulos, and P. Kokotovic, Nonlinear and Adaptive Control Design. New York: Wiley, 1995. 